\title{
Graph Models and GeoData Based Web Portal in Cargo Transportation
}

\author{
Leonids Novickis ${ }^{1}$, Svetlana Vinichenko ${ }^{2}$, Mihails Sotnichoks ${ }^{3}$, Andrejs Lesovskis ${ }^{4}$, Darja Amalitskaya ${ }^{5}$, \\ ${ }^{1,3,4}$ Riga Technical University, Latvia, ${ }^{2,5}$ Logitrans Consult Ou, Estonia
}

\begin{abstract}
The paper presents a Web Based Framework (WBF)/Web portal to support business processes in transport logistics.

The proposed portal has been developed using graph and multi-agent models and GeoData approach.

It is one of the main results of international project eINTERASIA supported by the Seventh Framework Program of the European Commission.
\end{abstract}

Keywords - GeoData, graph models, intermodal transportation, transport logistics, Web Based Framework, web services, web portal.

\section{CuRrent State AND Related Projects}

Intermodal freight transportation involves the use of two or more modes of transportation in a closely linked network for the seamless movement of goods. It is typically associated with containerization or, in more general terms, the transport of goods involving direct transfer of equipment between modes without any handling of transported goods [1].

The advantages of intermodal transport attract more and more enterprises to integrate it into their logistical strategies. As a result, the intermodal traffic continues to grow. The enterprises are in a way of optimizing their supply chain structure to benefit both from the transport logistics services and from the improved performance of economic activities as a whole. Generally, intermodal logistics will profit the enterprises on the following aspects [2]:

1) Door-to-door transport is the most desirable objective for the enterprises in their logistical organization and it is marked as one of the core characteristics of intermodal transport;

2) Transport cost reduction is a sought after objective of the enterprises. With the integration of water and rail transport, the intermodal transport in freight corridors (medium or long distance) has lower transport costs than the road transport, giving the privileges to be integrated into enterprises' supply-chain;

3) Environmental impact requirements are the requirements imposed on the transport and logistic service sector to reduce the society's cost (external cost). Thus, intermodal transport is a solution to shift traffic from the heavy loaded road network to the environmentally friendly transport modes (rails, waterways) without losing the convenience for its users. The costs caused by the congestion and pollution (including the noise) can be dramatically reduced through the implementation of intermodal logistics that is beneficial both for the enterprises and society;

4) Value-added services are appreciated by the enterprises facilitating the integration of production/consumption procedures through optimal logistical chain management. These services can easily be provided in the intermodal hubs (ports, terminals or platforms) using the opportunities of modal transfer breaks of intermodal transport processes. The value-added services would provide strategic, financial and organizational advantages for the enterprises.

Innovative IT solutions make it possible for the enterprises to organize their logistics and integrate intermodal transport into their supply chain management [3].

Transportation service providers that grew through acquisitions have multiple operating processes, multiple IT platforms and predominantly manual processes. At the same time, those providers often lack global visibility and are poorly integrated with suppliers and customers. Logistic organizations currently struggle with IT infrastructures made up of older platforms and multiple integration points. The transport logistics segment is characterized by a very high competition and especially strong pressure from logistics giants. Logistics giants with their international networks have significant advantages over small and medium-sized enterprises (SMEs). For individual SMEs it is difficult to solve the problems alone.

However, by co-operating, they can keep relatively big segment of the world transport logistics market.

SME actors have to cooperate and develop collaborative frameworks [4].

To achieve a more streamlined and agile "future state", most logistics companies must undergo technology-based transformation [5].

It is clear that in the future Internet-based solutions will revolutionize the world of SMEs that have to deal with transport logistics problems by creating a new platform of business and revenue opportunities [6].

Several research projects supported by the EC (e.g., BOPCOM, iCARGO, BPR Logistics, EUROBORDER, INFOLOG and INTERSPORT) and world-leading companies deal with software related to applications of logistics services and supply chain management. Examples are: IBS, i2 Technologies, FWL Technologies and SAP. Investigations of the design of logistics services (www.elitelog.com and www.miebach.com), and m-logistics developed to support the life cycle of goods (www.cals.nato.be) as well as e-forwarding (shercargo.ru, integprog.ru) are others. An analysis of solutions shows that collaborative environments in logistics and transport are mostly based on web and mobile platforms. These are becoming part of the corporate communication infrastructure. Recent progress in intelligent technologies 
(e.g., knowledge management and agent-based theory) has led to the development of so-called intelligent transport \& logistics systems, i.e., the TELETRUCK system, SPHERE, DIAL, TRANSIMS, and MALT [6]-[9].

An analysis of the different approaches taken by these systems shows that the Internet-based interoperability of intelligent technologies with modelling is the most promising combination of solutions that can be used to create the next generation of intelligent business frameworks and portals for transport logistics services.

At the moment, IT-based applications in transport logistics do not use the mechanisms of united access to heterogeneous e-resources (websites, databases, portals etc.) for facilitating a business process.

The paper is devoted to the development of software demonstrator of Web-based Framework (WBF)/Web portal, using graph models, GeoData, and intelligent multi-agent model.

\section{CONCEPT AND IMPLEMENTATION}

\section{A. Main Functions}

The portal www.einterasia.com allows users to organize collaborative work between representatives of two target groups: Transport and Cargo (Fig. 1).

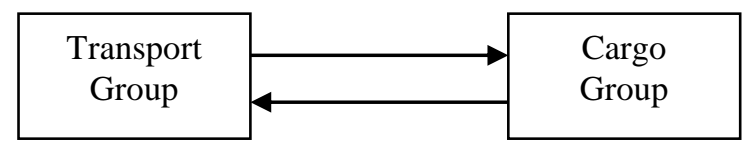

Fig. 1. Collaboration between two target groups.

For the group of cargo owners (exporters/ importers, brokers, traders) the portal enables:

- To calculate the transportation costs for cargo delivery from the place of purchase to the point of destination in accordance with INCOTERMS 2010;

- To calculate the transit time for cargo delivery to the point of destination;

- To choose the transport company that corresponds to the requirements of a cargo owner.

For companies from transport group (agents of shipping lines, railway freight agents, freight forwarders, road carriers, block train operators etc.) the portal enables:

- To involve new clients and to increase cargo turnover;

- To reduce workload of experts of a company to "a basket" offering to prospective clients rates of transportation which have already been calculated and saved in portal database, but not required and used because of some reasons;

- To increase profitability of a business using advanced Internet-based technologies;

- Apart from this, both groups of users can receive "Useful information" about transport logistics and related areas:

- Services of shipping lines and block trains;

- Education and Training;

○ IT and mobile solutions.

\section{B. Technological Solutions}

In order to achieve these functionalities, it was necessary to expose all the available e-resources as services. The integration was implemented following the principles of Service-Oriented Architecture (SOA).

There are many standalone research Internet portals and websites, which offer different scientific and technological information. WBF combines all those e-resources in one portal. The main benefit of WBF for the users and providers is that they can have a single access point to the combined information. From a technological point of view, the main objective is to create the best communication between WBF and separate websites and portals. In connection with this, web services are used as communications - endpoints. They can be implemented using different kinds of software technologies: for example, .NET, Java, PHP and others.

On the basis of web services, API (Application Program Interface) is being developed. With the API the information transfer between separate portals/web sites and WBF can be interchanged. For the hosting of an API two alternatives will be investigated (Fig. 2):

Alternative 1: An API is integrated within WBF. In this case separate e-resources will play the role of a Client.

Alternative 2: Each e-resource (web portal) offers its own API, whereas the WBF works as a Client.

Both variants have advantages and disadvantages, which were analyzed and discussed.

a) Alternative 1:

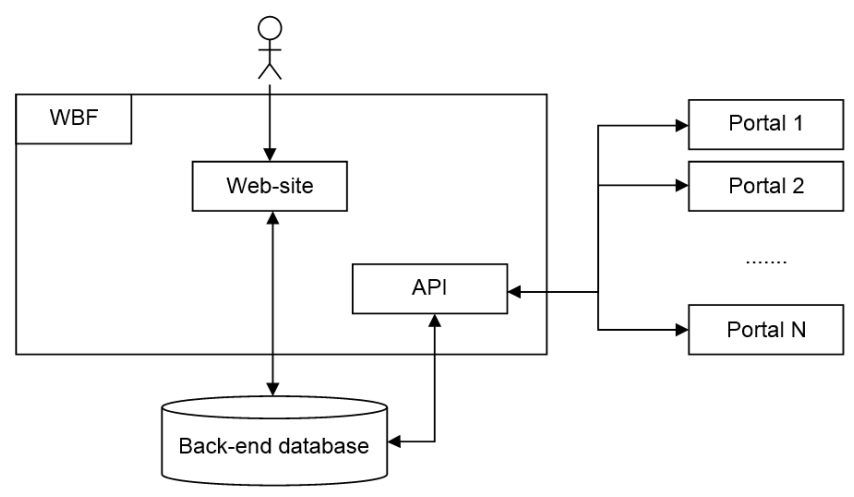

b) Alternative 2:

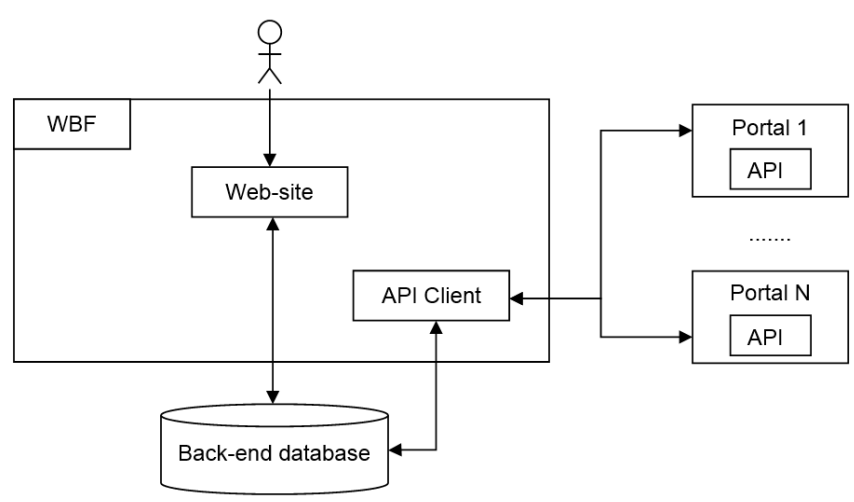

Fig. 2. Two alternatives of integration. 
eINTERASIA WBF always grants access to the latest version of its software that is maintained on a dedicated server (s). A user needs only a modern web browser and an Internet access.

The $1^{\text {st }}$ alternative was selected for implementation of eINTERASIA WBF.

Service-Oriented Architecture [10] is a paradigm for organizing and utilizing distributed capabilities that may be under the control of different ownership domains and implemented using various technology stacks. In general, entities (people or organizations) create capabilities to solve or support a solution for the problem they face in the course of their business. In our case, actors from cargo and transport groups collaborate to solve the problem of effective cargo transportation and increasing business profitability.

There are multiple benefits of the SOA integration approach and the most important of them is [11]: "becoming more agile".

SOA enables business partners to respond quickly to new business conditions and to develop new capabilities.

On a technical level, Web services can be implemented in various ways, for instance, "big" web services and "RESTful" web services.

"Big" web services use XML messages that follow the Simple Object Access Protocol (SOAP) standard. XML language is used to define the message architecture and message formats. A major design goal for SOAP is simplicity and extensibility [12].

REST defines a set of architectural principles by which web services can be designed. They focus on a system resources, including how resource states are addressed and transferred over HTTP by a wide range of client written in different languages [13].

After analyzing both SOAP and REST, it has been concluded that both approaches work and both have advantages and disadvantages to interfacing to web services.

In our case (enterprises level WBF), SOAP is a better choice for several reasons:

- SOAP supports different protocols and technologies, including WSDL, XSDs, WS-addressing;

- SOAP was designed to handle distributed computing environments;

- SOAP is well suited for intermediated services;

- SOAP has a large number of supporting standards for security, reliability, transactions etc.

That is why SOAP approach has been selected for WBF implementation.

SOAP web services can be successfully used to integrate new functionality and functionality from the existing systems into the portal.

One of the demonstration scenarios illustrates the use of SOAP web services to ensure portlet functionality and interportlet communications. Demonstration was based on elogmar-M/ eInterasia portal version. Weather service and corresponding portlet, which gets data about selected points of departure and arrival and shows weather forecast for them, have been tested (Fig. 3).

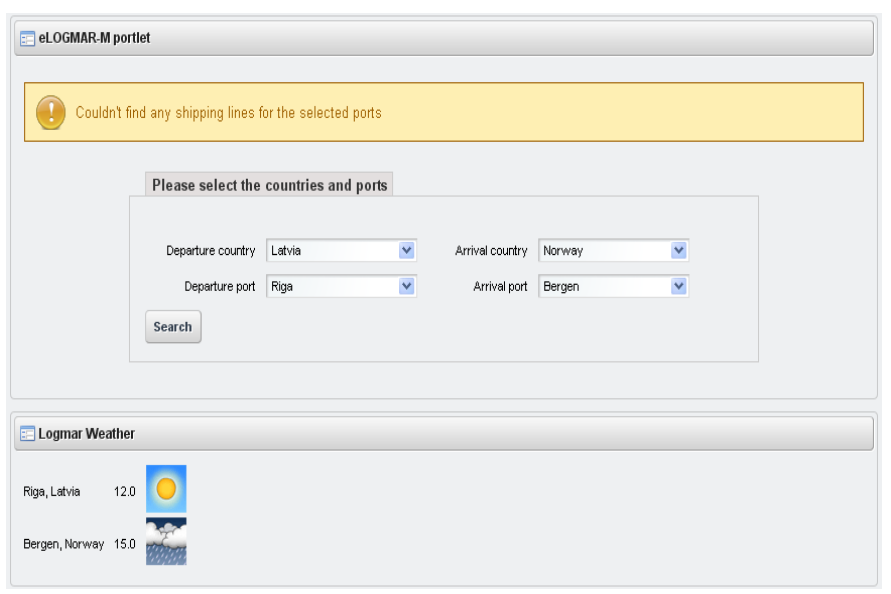

Fig. 3. Inter-portlet communication between shipping line services and weather portlets.

Special web service was created to communicate with a railway portal and to integrate the calculation of block train freight prices into www.einterasia.com tariff calculation algorithms (Fig. 4).

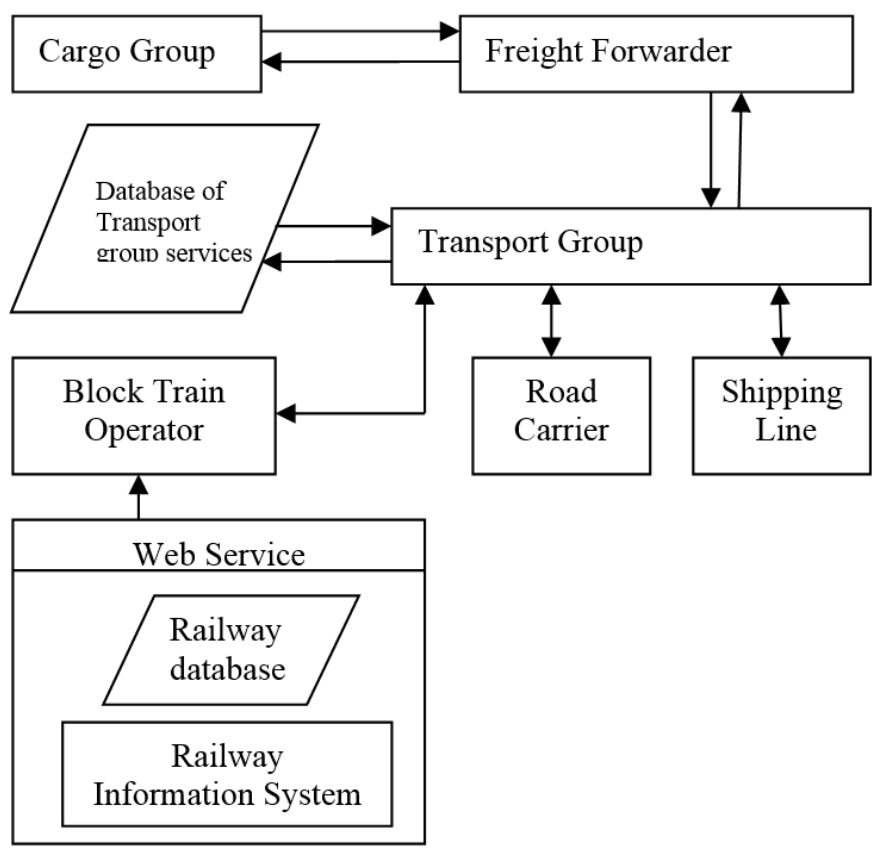

Fig. 4. Sample of the use of web service.

\section{ALGORITHM OF INTERMODAL CONTAINER TRANSPORTATION}

Web portal offers intermodal container transportation using several modes (sea, rail, or road) without any handling of the freight itself when changing the mode.

The request processing algorithm for intermodal transportation uses Google Map Web services and includes the following main steps:

1. For each departure and destination point (ports, railway stations, cities/warehouses) a portal administrator has to define the areas around them by selecting a radius and fixing it. Firstly, a portal administrator has to define GPS coordinates (Latitude - Lat., Longitude - Long.) for all points of departure 
and destination by clicking on the Google Map. Then he must define a radius and areas around points of departure and destination (as can be seen in Fig. 5).

2. Actors from the Transport Group using a set of interface forms enter to the portal database transportation tariffs and services for a selected freight route.

Three interface forms for direct container transportation between points of departure and destination are foreseen: seasea, rail-rail, and road-road.

3. Actors from the Cargo Group can create on-line transport request (Fig. 7) and receive data on cargo transportation rates and estimated transit time using an option "Freight cost calculation".

If corresponding request cannot be provided with just one transportation mode (sea-sea, rail-rail or road-road), a processing procedure for intermodal transportation is applied.

The procedure is based on the use of graph model of possible transportation routes between the points of departure and destination [14].

Intermodal transportation includes several services (freight route, tariff rates, expected transit time) which are connected by GPS coordinates of point of departure and destination.

Taking into account defined radius, points are united into one sector.

Each sector represents a node of route graph (Fig. 6).

4. The procedure searches for the ways on graph model between points of departure and destination defined in on-line transport request.

Restriction: nodes of a graph are included only one time in the way.

5. Total transportation rates and estimated transit time are calculated. Data of separate services are summed up.

As a result of calculations, all possible ways and their sections (lists of nodes) of transportation are presented in a table (Fig. 8).

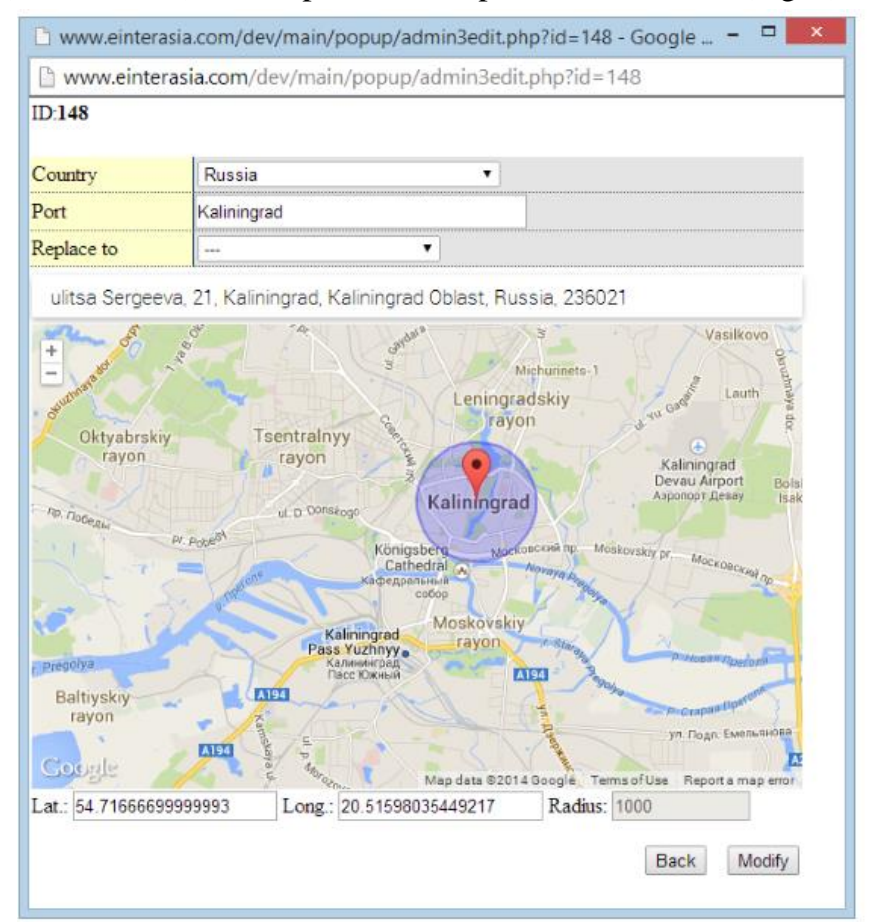

Fig. 5. Radius and areas around points of departure and destination.

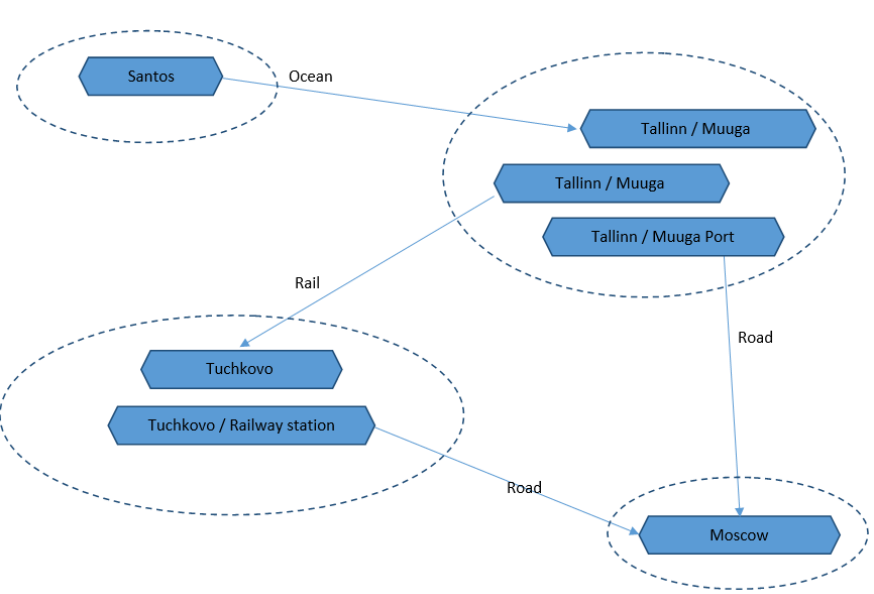

Fig. 6. Graph of intermodal transportation route (sample: Santos Tallinn/Muuga - Moscow).

Stage 1: Definition of transport route

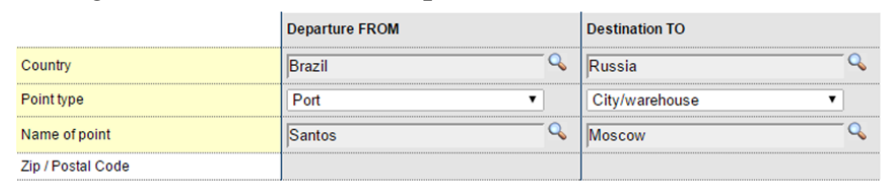

Stage 2: Cargo description
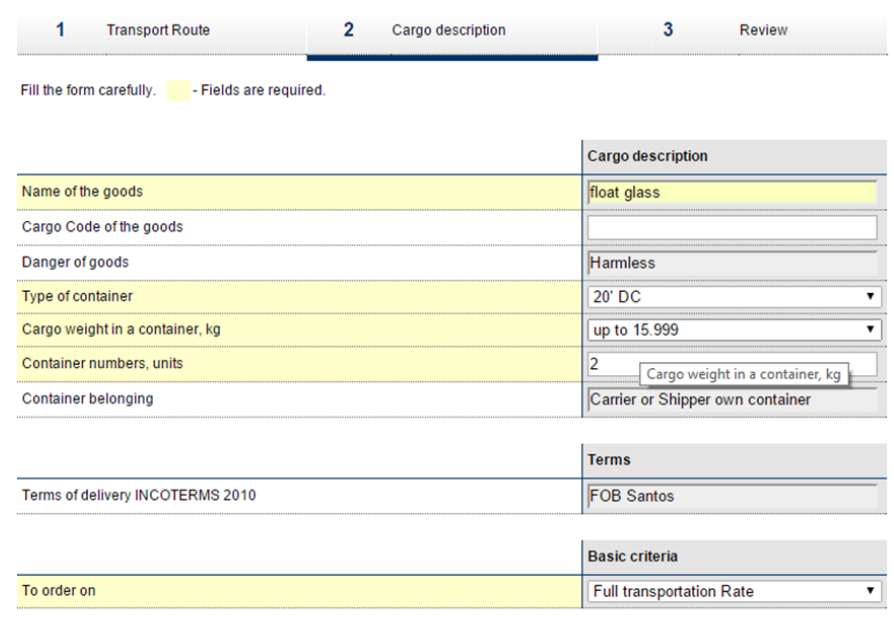

Fig. 7. On-line transport request. 


\begin{tabular}{|c|c|c|c|c|c|c|c|}
\hline Santos $\rightarrow$ Tallinn / Muuga $\rightarrow I$ & oscow & & & & & & \\
\hline \multirow[t]{2}{*}{ Transport company } & \multirow[t]{2}{*}{ Transport Route } & \multirow[t]{2}{*}{ Currency } & \multicolumn{2}{|c|}{$\begin{array}{c}\text { Full } \\
\text { Transportation } \\
\text { Rate }\end{array}$} & \multirow{2}{*}{$\begin{array}{c}\text { Validity } \\
\text { date }\end{array}$} & \multirow{2}{*}{$\begin{array}{l}\text { Expected } \\
\text { transit } \\
\text { time, } \\
\text { days }\end{array}$} & \multirow[t]{2}{*}{ Type } \\
\hline & & & $\begin{array}{c}\text { One } \\
\text { container }\end{array}$ & $\begin{array}{c}\text { Cargo } \\
\text { LOT }\end{array}$ & & & \\
\hline Forwarder / Logistic Company 1002 & Santos - Tallinn / Muuga & USD & 1889 & 3778 & 10.03 .2015 & 28 & Ocean \\
\hline Forwarder / Logistic Company 1002 & Tallinn / Muuga Port - Moscow & EUR & 1450 & 2900 & 31.03 .2015 & 3 & Road \\
\hline \multicolumn{3}{|r|}{ Total USD: } & 3519 & 7038 & & 31 & \\
\hline
\end{tabular}

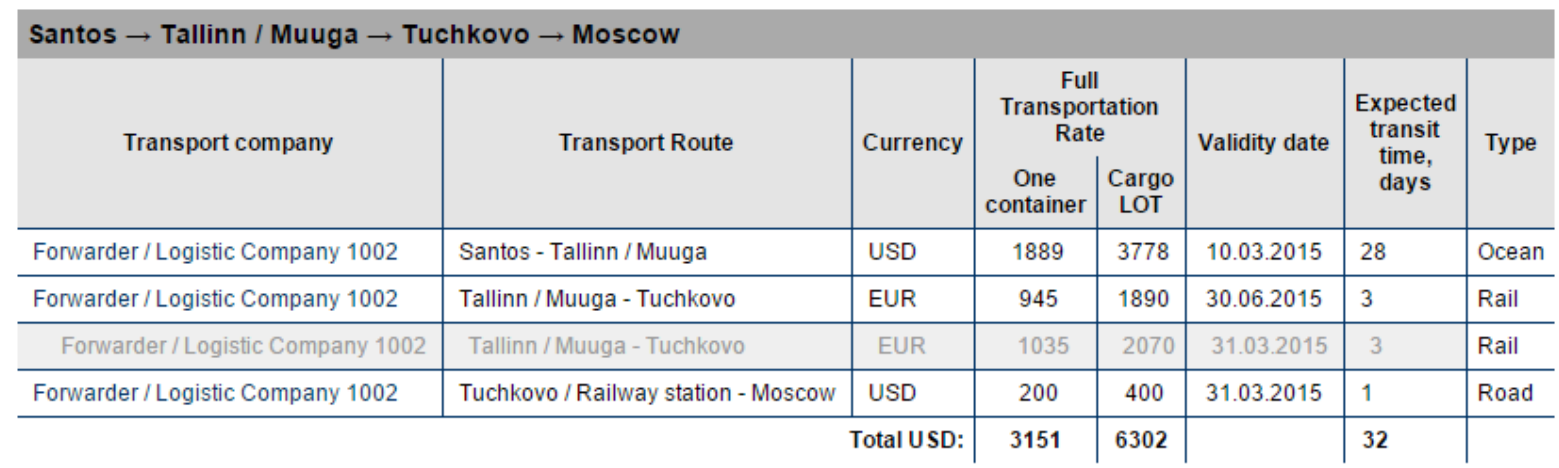

Fig. 8. The results of processing of on-line request.

\section{CONCLUSION}

To improve the business processes in the area of transport logistics, Web-Based Collaborative Framework (web portal) has been developed (www.einterasia.com).

The web portal is based on the use of graph models of cargo transportation routes and GeoData and represents the modified version of eLOGMAR portal www.elogmar.eu [9] that was adapted to the specific needs and conditions of the target region of Central Asia [15].

Cargo auction mode in the web portal is based on a multiagent model that has already been successfully used in insurance area [16]. Cargo auction model is presented in the paper "Multi-Agent Based Cargo Auction" (the authors are J. Grundspenkis and E. Lavendelis) of the same Scientific Journal of Riga Technical University.

Therefore, in comparison with other related projects the web portal is based on synergy of several innovative components: advanced web technologies and graph and intelligent multi-agent models. The proposed approach allows extending a set of portal functions and improving the quality of provided services.

During the $1^{\text {st }}$ stage of the portal validation, potential end users (volunteers) were involved in the process. They were asked to fill in a special questionnaire for the estimation of the quality of portal functional modes (to enter transportation tariffs and services, to calculate cargo transportation rates and estimated transit time, useful information in education \& training and IT \& Mobile solutions, cargo auction) and user interface in terms of simplicity and clarity of the portal usage. Five-point scale (1-5) has been applied: "5" - excellent, "4" good, "3" - average, "2" - poor, "1" - very poor.

Potential users from the EU and Central Asia were involved in the process of validation and estimation: in total 18 organizations have been presented. The results of users' feedback show that in general the quality of portal functionality as well as user interface has been estimated positively, but some remarks have been formulated:

- portal functionality ( mark " 5 " $-60 \%$, mark " 4 " - 40\%),

- user interface (mark " 5 " - 55\%, mark " 4 " - 45\%),

- option "Cargo auction" is the most complicate mode from the point of view of user interface.

Feedback results will be taken into account by portal developers during the $2^{\text {nd }}$ stage (June-July 2015) of validation and estimation.

The future plans include further commercialization of web portal and its exploitation in Central Asia and other regions.

\section{ACKNOWLEDGEMENT}

The research has been partly supported by the project eINTERASIA "ICT Transfer Concept for Adaptation, Dissemination and Local Exploitation of European Research Results in Central Asian Countries", grant agreement No. 600680 of the Seventh Framework Program Theme ICT9.10.3: International Partnership Building and Support to Dialogues for Specific International Cooperation Actions CP-SICA-INFSO.

\section{REFERENCES}

[1] OECD Publishing, Intermodal Freight Transport: Institutional aspects, 2011.

[2] Bontekoning, Y.M., "The importance of new-generation freight terminals for intermodal transport," 2010. http://dx.doi.org/10.1002/atr.5670340305

[3] Marchal, J., Macharis, C., Zhang, Z., Pekin, E., "The Added Value of Intermodal Transport for the Logistical Organization of Enterprises: A Study on the Opportunities and Bottlenecks," [Online]. Available: http://orbi.ulg.ac.be 
[4] Novickis, L., Lesovskis, A., Mitasiunas, A, "Technology Transfer Model and Web-Based Solutions for Transport Logistics Services providers," in Proc. of the European Computing Conf., ECC'2011, WSEAS Press, Paris, pp. 132-136, 2011.

[5] Charalabidis, Y., Gionis, G., Hermann, K.M., C.Matinez, C. (eds.) European Commission, Enterprise Interoperability. Research Roadmap. Version 5.0., 2008.

[6] The Future of the Internet Conf., A Conf. held under the Czech Presidency of the EU, Prague, May 11-13, 2009.

[7] "SPHERE: Small and medium sized Ports with Harmonized Effective Re-engineering Processes," EU funded project under Transport Programme, 1998-2000.

[8] Schoenhof, P., "International brokerage of Freight Holding Equipment," in Scientific Proc. of the Project eLOGMAR-M, JUMI, Riga, pp. 103106, 2006

[9] Bluemel, E., Novitski, L., Strassburger, S. (eds.), Scientific Proc. of the Project eLOGMAR-M. JUMI, Riga, 2006.

[10] Rosen, M., Lublinsky, B., Smith, K., and Balcer, J., Applied SOA: Service-Oriented Architecture and Design strategies. Wiley, p. 696 2008.

[11] W3C Working Group. Web Services Architecture. [Online]. Available: http://www.w3.org/TR/ws-arch/

[12] W3C. SOAP Specification. [Online]. Available: http://www.w3.org/TR/soap/

[13] Daigneau, R., Service Design Patterns: Fundamental Design Solutions for SOAP/WSDL and RESTful Web Services. Addision-Wesley Professional, p. 352, 2011.

[14] Aldous, J.M., Wilson, R.J., Graphs and Applications: An Inroductory Approach. Springer-Verlag, London, p. 444, 2000, ISBN I-85233259-X

[15] Council of the European Union, "The European Union and Central Asia New Partnership in Action," [Online]. Available: http://ec.europa.eu/external_relations/central_asia/docs/2010_strategy_e u_centralasia_en.pdf

[16] Lavendels, E., Grunspenkis, J., "Multi-Agent Auction Based Simulation Tool for an Insurance policy Market," in Scientific J. of Riga Technical University. Applied Computer Systems, 2014/15, RTU Press, pp. 5-13, 2014

Leonids Novickis is the Head of the Division of Software Engineering. He obtained Dr. sc. ing. degree in 1980 and Dr. habil. sc. ing. degree in 1990 from the Latvian Academy of Sciences. Since 1994, he has been regularly involved in different EU-funded projects: AMCAI (INCO COPERNICUS, 1995-1997) WP leader; DAMAC-HP (INCO2, 1998-2000), BALTPORTS-IT (FP5,
2001-2003), eLOGMAR-M (FP6, 2004-2006) - scientific coordinator; IST4Balt (FP6, 2004-2007), UNITE (FP6, 2006-2008) and BONITA (INTERREG, 2008-2012) - RTU coordinator; LOGIS, LOGIS-Mobile and SocSimNet (Leonardo da Vinci) - partner. He was an independent expert of IST and Research for SMEs in FP6, FP7. He is a corresponding member of the Latvian Academy of Sciences. His research fields include applied software system development, business process modelling, eLogistics, international cooperation, web-based applications. $\mathrm{He}$ is the coordinator of FP7 eINTERASIA project.

E-mail: lnovickis@gmail.com

Svetlana Vinichenko She is the Director of transport forwarding company Logitrans Consult (Estonia). She graduated from Odessa Institute of Marine Engineers as a Specialist in Marine Business Organization and Management. She took part in several EU projects: BALTPORTS-IT (partner), eLogmar-M (work package leader), eINTERASIA (work package leader)

Her areas of interests are: Internet-based business solutions, modeling of business processes in intermodal cargo transportation.

E-mail: svetlana.vinichenko@mail.com

Mihails Sotnichoks He obtained Mag.sc.comp. degree from Riga Technical University, participated in several EU projects as a Software Engineer: IST4Balt, UNITE and eLOGMAR-M (FP6), BONITA (BSR INTEREG Programme). Currently he is involved in the eINTERASIA project (FP7) as a Leader of Software Development Group. His activities are related to the development of web-based applied solutions.

E-mail:msv@rtu.lv

Darja Amalitskaya She graduated the Estonian Maritime Academy and obtained a Bachelor Degree in Port and Shipping Management and continued with Master's studies at Tallinn University of Technology, the Department of Logistics with focus on supply chain engineering. She participated as a Logistician in the EU project eLOGMAR-M (FP6). Currently she is involved in the eINTERASIA project (FP7) as a Logistician to develop transport and logistics solutions.

E-mail: darja@logitrans.ee

Andrejs Lesovskis is a Doctoral Student at Riga Technical University, the Faculty of Computer Science and Information Technology. He obtained the Master Degree in Computer Science and Information Technology at Riga Technical University in 2009. His research areas are e-Learning and Semantic Web. He works as a Researcher at Riga Technical University.

E-mail: andreyl@inbox.lv 